\title{
Simulation numérique de l'écoulement laminaire induit dans des cuves agitées générées par les mobiles de proximité de type mono et double vis à profils simple et modifié
}

\author{
Zied Driss ${ }^{\mathrm{a}}$, Ghazi Bouzgarrou, Hedi Kchaou et Mohamed Salah Abid \\ Laboratoire des Systèmes Electromécaniques, École Nationale d'Ingénieurs de Sfax, Université de Sfax, BP 1173, \\ Route de Soukra, 3038 Sfax, Tunisie
}

Reçu le 10 juin 2010, accepté le 14 décembre 2010

\begin{abstract}
Résumé - Dans cet article, une étude comparative entre les caractéristiques hydrodynamiques de l'écoulement laminaire généré en cuves agitées par différents mobiles de proximité de type mono et double vis avec profils simple et modifié a été menée en utilisant la simulation numérique. Cette étude a été abordée à l'aide d'un code spécifique de dynamique des fluides numérique (CFD), basé sur la résolution des équations de Navier-Stokes par la méthode des volumes finis. Les résultats numériques obtenus montrent bien l'influence de la forme du mobile d'agitation sur le comportement de l'écoulement au sein de la cuve. En effet, on remarque que l'utilisation d'une vis à profil modifié favorise un champ de vitesse plus actif que dans le cas des configurations à profil simple. Par ailleurs, et dans le cas d'une double vis, on note que l'effet de la dissipation visqueuse et du pompage est nettement plus prépondérant, présentant ainsi une meilleure efficacité de pompage et de consommation énergétique. La comparaison de nos résultats numériques avec les résultats expérimentaux tirés de la littérature montre une bonne adéquation, ce qui prouve la validité de la méthode d'analyse adoptée.
\end{abstract}

Mots clés : Modélisation / code CFD / volume fini / cuve agitée / mobile de proximité / vis d'Archimède

\begin{abstract}
Computer simulation of the laminar flow in stirred tanks generated by the proximity impellers of a mono and double screws type with simple and modified profiles. In this paper, the hydrodynamic characteristics of stirred tanks equipped with a mono and double screws with both classic and modified profiles have been numerically investigated. The computer simulations are conducted within a computational fluid dynamic (CFD) code, based on resolution of the Navier-Stokes equations with a finite volume discretization. The numerical results showed that the velocity field is more efficient with the modified screw profile than the one with a simple profile. In the case of the double screw, the importance of viscous dissipation and pumping has been assessed. Also, the pumping and the energetic efficiency reach the highest values. The good agreement between the numerical results and the experimental data confirm the validity of the analysis method.
\end{abstract}

Key words: Modelling / CFD code / finite volume / stirred tank / proximity impeller / Archimedes screw

\section{Introduction}

L'agitation mécanique intervient dans la plupart des procédés industriels. Son domaine d'application est très étendu, depuis le mélange des fluides miscibles jusqu'à la fabrication des produits pharmaceutiques. La connaissance d'un maximum d'informations précises sur les structures fines des écoulements, générés par les mobiles d'agitation, est nécessaire pour la mise au point et l'optimisation de diverses opérations industrielles. Une telle analyse

\footnotetext{
a Auteur pour correspondance: Zied.Driss@enis.rnu.tn
}

est de plus en plus demandée car les coûts énergétiques inhérents aux opérations d'agitation affectent d'une part la qualité du produit obtenu et d'autre part la tendance mondiale à l'économie d'énergie [1]. Pour répondre aux souhaits des industriels, les concepteurs de matériels d'agitation doivent fournir des connaissances fiables sur les performances de leurs produits. En effet, le choix de la taille et de la géométrie de l'agitateur est une étape essentielle dans la recherche du système le mieux adapté à l'opération de mélange et d'agitation réalisée. Aujourd'hui, grâce au développement des outils de simulation 


\section{Nomenclature}

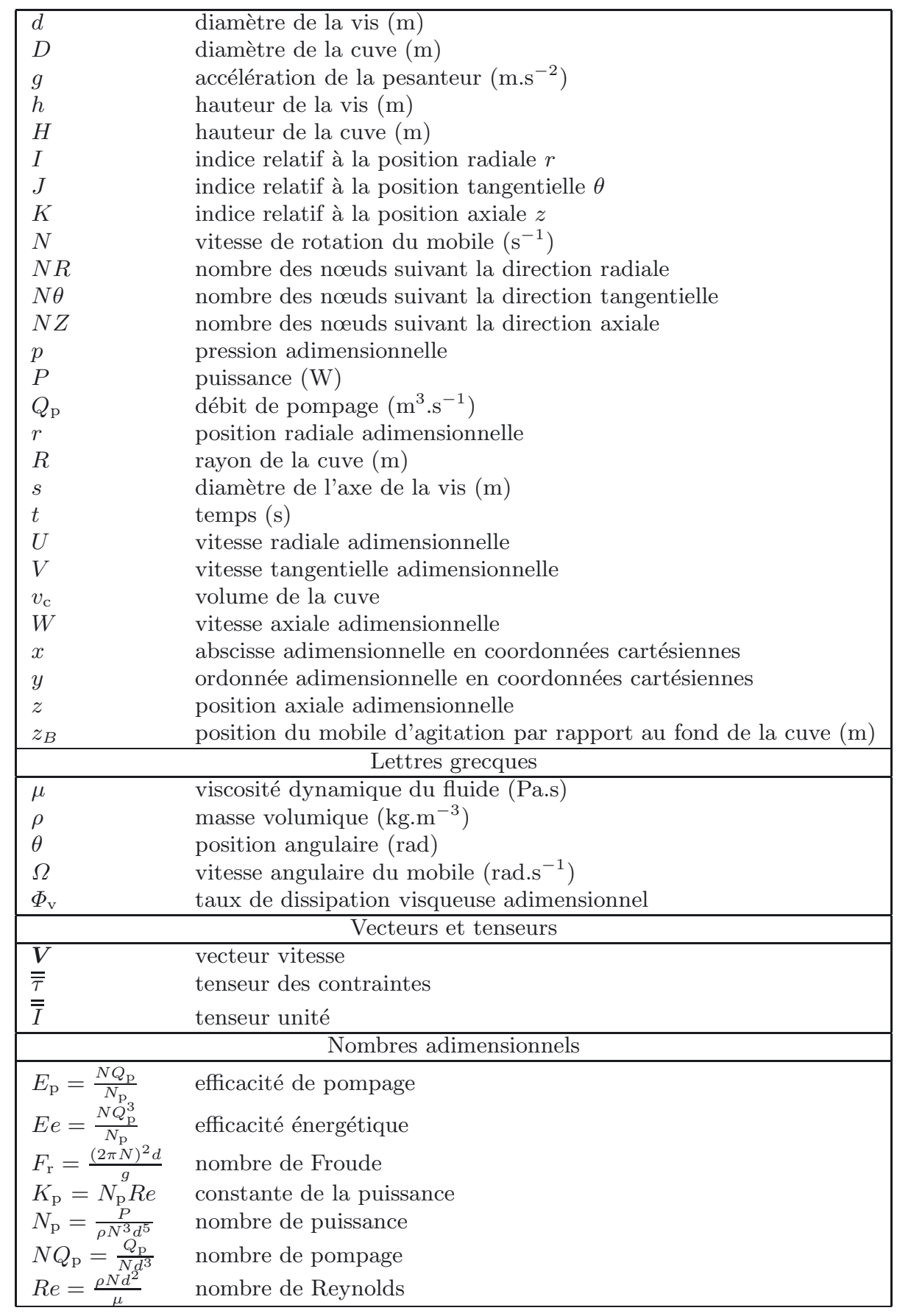

numérique et les techniques de mesure et d'acquisition, de nouvelles avancées technologiques ont été concrétisées tant au niveau expérimental que théorique $[2,3]$. Actuellement, la dynamique des fluides numérique (CFD) a atteint un degré de perfectionnement permettant la simulation des écoulements très complexes, impliquant des évolutions en régime dynamique et montre bien une complémentarité avec les travaux expérimentaux $[4,5]$. C'est dans ce cadre que se situe le présent travail dont le but est de chercher les différents paramètres géométriques qui influent directement sur les caractéristiques hydrodynamiques et les conditions opératoires d'agitation et de mélange. Généralement, l'opération de mélange doit assurer soit une action de pompage caractérisée par un écoulement axial ou radial ou une action de turbulence au voisinage des parois favorisant un cisaillement plus ou moins élevé. Dans cet article, on s'intéresse particulièrement aux mobiles de proximité, telles que les vis d'Archimède, qui se révèlent beaucoup plus performants pour mélanger les fluides fortement visqueux. Plusieurs travaux s'attachant à ces mobiles d'agitation ont été réalisés, on cite à titre indicatif, ceux de Nagata [1], Valette et al. [5], Delaplace et al. [6], Bertrand et al. [7], Bellhouse et al. [8], Rieger [9] et Rorres [10]. En effet, 
dans son travail expérimental, Nagata [1] s'est intéressé à la variation du nombre de puissance en fonction du nombre de Reynolds d'une cuve agitée munie d'une vis d'Archimède. Il a également étudié différentes configurations géométriques afin de choisir les mobiles d'agitation les plus performants lors de l'opération d'agitation et de mélange. Valette et al. [5] ont présenté une méthode d'interaction fluide-structure basée sur une approche monolithique eulérienne permettant d'étudier à différentes échelles les procédés de mélange sous leur aspect dispersif et distributif. Une approche macroscopique traitant de la résolution mécanique dans le procédé, où interviennent des outils tournants, est présentée, puis une approche microscopique modélisant la dispersion d'un agglomérat, et enfin une approche couplée via une théorie cinétique. Delaplace et al. [6] ont développé un modèle analytique pour calculer la puissance consommée au cours de l'opération de mélange en régime laminaire. Dans cette application, les auteurs se sont particulièrement intéressés à l'effet de la combinaison d'une vis et un ruban hélicoïdal. La comparaison des résultats trouvés avec les travaux expérimentaux tirés de la littérature a montré l'applicabilité du modèle analytique développé pour une grande gamme de configurations. Par ailleurs, les auteurs ont montré que le modèle est capable d'expliquer la différence avec les données rapportées de la littérature et de prédire la dépendance entre la puissance consommée avec les propriétés du fluide mélangé ainsi qu'avec les paramètres géométriques du système. Bertrand et al. [7] se sont intéressés à l'étude de l'écoulement dans une extrudeuse à vis. Une simulation numérique en 2D a été développée en régime transitoire dans le domaine balayé par les vis. Bellhouse et al. [8] ont étudié les performances des vis hélicoïdales insérées dans les membranes tubulaires. Ces auteurs ont montré qu'un tel système permet un bon mélange et minimise les effets de polarisation et de concentration. Rieger [9] s'est intéressé dans son travail à l'étude de l'effet du nombre de Reynolds sur l'efficacité de pompage d'un agitateur à vis. La dépendance du pompage par rapport au nombre de Reynolds lui a permis d'étendre ses résultats à d'autres agitateurs utilisés en régime turbulent. Rorres [10] a montré que la performance d'une vis d'Archimède est gouvernée par les paramètres géométriques externes et internes. Les paramètres externes tels que le rayon extérieur, la longueur et l'inclinaison sont déterminés par l'emplacement de la vis et de la quantité de fluide soulevée. Cependant, les paramètres internes tels que le rayon intérieur, le nombre des spires et leurs inclinaisons doivent être choisis pour pouvoir améliorer les performances de la vis. Dans son article, l'auteur a montré que le rayon intérieur ainsi que l'inclinaison des spires maximisent le volume soulevé dans un tour de la vis. Les valeurs des paramètres optimales trouvées sont comparées avec les valeurs utilisées par les architectes romains ainsi qu'avec les valeurs utilisées pour la conception des vis d'Archimède modernes.

De ce fait, étant donnée la complexité de la géométrie de ces mobiles, peu de travaux numériques se sont intéressés à la caractérisation de la structure hydrody-

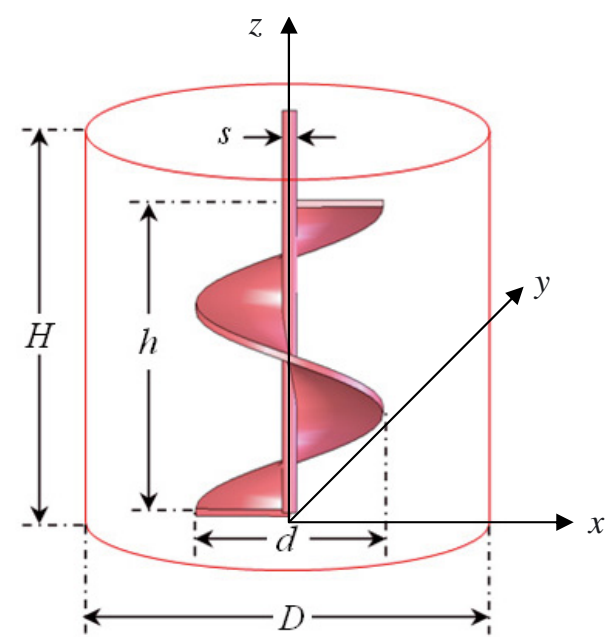

Fig. 1. Cuve agitée équipée d'une mono vis à profil simple.

Tableau 1. Paramètres géométriques.

\begin{tabular}{cc}
\hline Paramètres & Valeurs \\
\hline Diamètre de la cuve & $D=300 \mathrm{~mm}$ \\
Hauteur de la cuve & $H=300 \mathrm{~mm}$ \\
Diamètre de la vis & $d=190 \mathrm{~mm}$ \\
Hauteur de la vis & $h=285 \mathrm{~mm}$ \\
Diamètre de l'axe de la vis & $s=15 \mathrm{~mm}$ \\
Position de la vis & $z_{B}=15 \mathrm{~mm}$ \\
\hline
\end{tabular}

namique des cuves agitées munie d'une vis d'Archimède. Dans le présent travail et suite aux améliorations apportées à notre code CFD développé au sein de notre laboratoire $[11,12]$, on a réussi à simuler numériquement le comportement hydrodynamique de l'écoulement généré par ce type de mobile. Nous nous sommes intéressés également à une étude comparative de la structure hydrodynamique générée par de nouvelles configurations géométriques définies suite à l'adjonction d'une deuxième vis ou à la modification du profil de la vis.

\section{Système d'agitation à vis}

Dans cet article, on se propose d'étudier l'influence de la forme géométrique de différents types de mobiles de proximité sur la structure hydrodynamique des cuves agitées. La première application, à laquelle nous nous sommes intéressés, est constituée d'une cuve agitée munie d'une vis d'Archimède (Fig. 1). Ce mobile d'agitation appelé également mono vis à profil simple a été étudié expérimentalement par Nagata [1]. Dans ces conditions, les paramètres géométriques sont présentés dans le tableau 1.

Dans le présent travail, nous nous sommes intéressés également à de nouvelles configurations géométriques caractérisées par des vis à profils modifiés de type semicirculaire (Fig. 2b). Ces deux mobiles d'agitation sont également étudiés dans des configurations constituées d'une combinaison de double vis (Figs. 2c et d). En effet, en conservant les mêmes paramètres géométriques, l'adjonction d'une deuxième vis placée à $180^{\circ}$ par rapport 


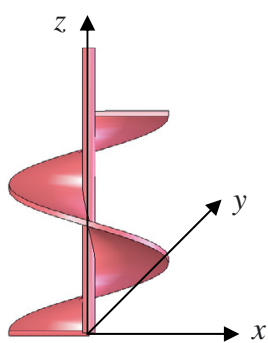

(a) Mono vis à profil simple

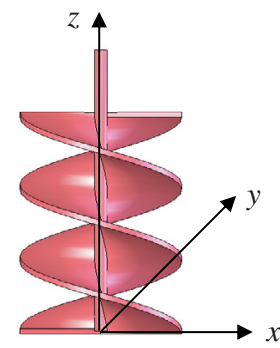

(c) Double vis à profil simple
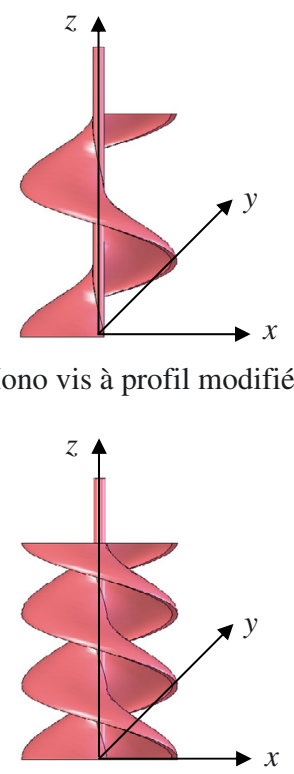

(d) Double vis à profil modifié (b) Mono vis à profil modifié

Fig. 2. Mobiles d'agitation.

à la première permet de concevoir de nouveaux mobiles d'agitation permettant éventuellement l'amélioration de la qualité du mélange. Dans ces conditions, les plans verticaux passant par l'axe de rotation du mobile forment ainsi des plans antisymétriques.

\section{Formulation mathématique}

Le code CFD développé nous a permis de caractériser la structure hydrodynamique dans une cuve mécaniquement agitée par les mobiles de proximité de type mono et double vis à profils simple et modifié. Ce code est fondé sur la résolution des équations de Navier Stokes et de continuité $[11,12]$.

Le principe de conservation de la masse annonce que la vitesse d'accumulation de la matière est égale à la différence entre le débit d'entrée de matière et le débit de sortie. En supposant que le flux est stationnaire et que le fluide est incompressible, c'est-à-dire à masse volumique constante, l'équation de continuité s'écrit :

$$
\operatorname{div} \boldsymbol{V}=0
$$

L'expression de la conservation de la masse dans un repère fixe est analogue à celle obtenue en repère mobile. Cependant, dans l'expression de l'équation de quantité de mouvement, on remarque qu'en repère mobile, il apparaît deux termes supplémentaires à savoir l'accélération de Coriolis $\gamma_{\mathrm{C}}$ et l'accélération centrifuge $\gamma_{\mathrm{c}}$ :

$$
\begin{aligned}
& \gamma_{\mathrm{C}}=2 \Omega \wedge \boldsymbol{V} \\
& \gamma_{\mathrm{c}}=-\Omega^{2} \boldsymbol{r}
\end{aligned}
$$

avec :

$$
\Omega=2 \pi N
$$

De ce fait, l'équation de la quantité de mouvement s'écrit :

$$
\frac{\partial(\rho \boldsymbol{V})}{\partial t}+\operatorname{div}(\rho \boldsymbol{V} \otimes \boldsymbol{V}+p \overline{\bar{I}})=\operatorname{div}(\overline{\bar{\tau}})+\rho\left(\Omega^{2} \boldsymbol{r}-2 \boldsymbol{\Omega} \wedge \boldsymbol{V}\right)
$$

Le comportement newtonien est caractérisé par la loi de proportionnalité suivante :

$$
\overline{\bar{\tau}}=2 \mu \overline{\bar{D}}
$$

En régime laminaire, les variables du problème sont la pression $p$ et les trois composantes du vecteur vitesse $U, V$ et $W$ obtenues en faisant la projection suivant les trois axes d'un système tridimensionnel à coordonnées cylindriques $(r, \theta, z)$. Notre code de calcul est applicable aussi bien aux problèmes des écoulements bidimensionnels que tridimensionnels et facilite le traitement des conditions aux limites. La méthode de discrétisation aux volumes finis, décrite en détail par Patankar [13], est utilisée puisqu'elle assure la continuité des flux entre les nœuds. La technique consiste à découper le domaine de calcul en volumes élémentaires entourant chaque nœud du maillage. La discrétisation spatiale est obtenue en suivant une procédure d'interpolation au schéma hybride. Quant à la discrétisation temporelle, la formulation implicite est adoptée. L'équation de transport à résoudre est alors intégrée sur chacun de ces volumes exprimant le bilan des flux de la variable transportable. L'intégration dans le temps est entreprise en utilisant le schéma aux directions alternées de Douglas-Gunn [14]. Le couplage pression-vitesse repose sur l'algorithme Simple [13]. Dans le présent travail, l'ordinateur utilisé est un Pentium (R) 4 équipé d'un processeur 2,8 GHz et d'une RAM 1 Go. De ce fait, le temps de calcul est de $15 \mathrm{~h}$ environ. Ce temps dépend également du maillage utilisé, des conditions initiales, du nombre de Reynolds $R e$ et de la précision souhaitée.

\section{Méthode d'adaptation du maillage}

Pour le maillage du volume de contrôle, on a appliqué la méthode d'adaptation du maillage du domaine fluide au maillage préalablement construit sur l'interface séparant le domaine fluide du domaine solide. Cette méthode présente un grand intérêt puisqu'elle permet de traiter le problème des géométries complexes. Elle consiste à générer le maillage du volume de contrôle à partir des nœuds construits préalablement sur l'interface séparant le domaine fluide du domaine solide de l'agitateur. Il s'agit de créer un maillage adaptable à la forme géométrique du mobile d'agitation (Fig. 3). La première étape commence par la construction de la géométrie du mobile d'agitation à l'aide d'un logiciel de conception assistée par ordinateur (CAO) tel que SOLIDWORKS. Cette forme géométrique est maillée en éléments-finis pour créer une liste de nœuds appartenant à l'interface fluide-solide. Ce maillage est réalisé à l'aide du logiciel de 
Maillage initial du domaine fluide Maillage modifié Maillage adapté à la forme de la vis à profil semi-circulaire
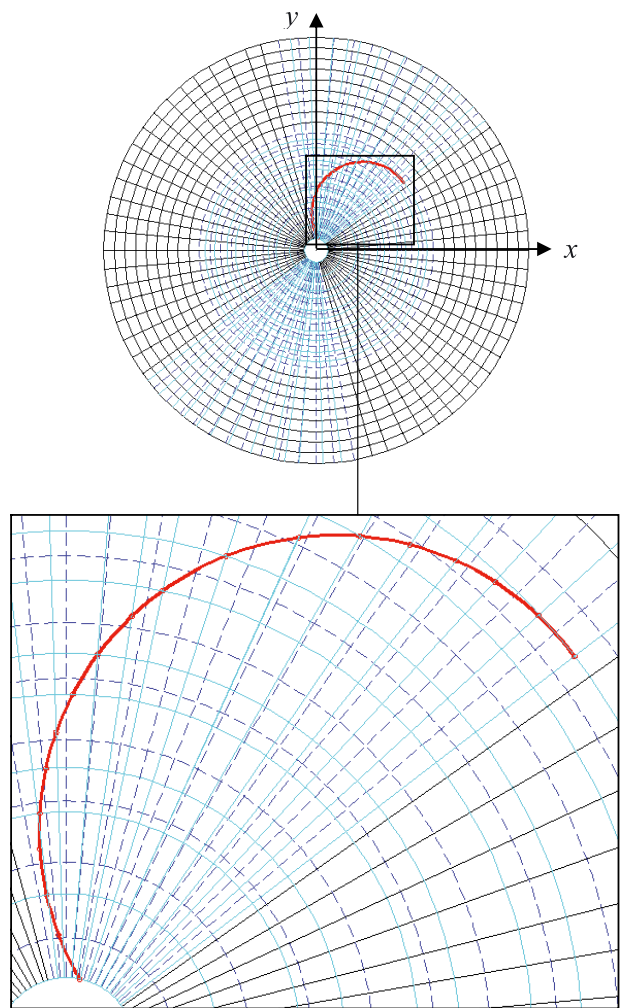

Fig. 3. Coupe selon le plan horizontal du maillage adapté.

dynamique des structures numériques (CSD) COSMOS. Ce logiciel permet de créer un fichier contenant à la fois le numéro et les coordonnées spatiales des nœuds. Le fichier de données créé est ensuite introduit dans notre interface graphique pour compléter le maillage du domaine fluide. La méthode adoptée consiste à une génération du maillage du volume de contrôle à partir des premiers nouds déjà créés sur l'interface fluide-structure. Cette étape consiste à définir le maillage dans le volume de la cuve tout en tenant compte des nœuds déjà créés sur l'interface fluidestructure. Dans notre application, le domaine de calcul considéré contient 108000 mailles. L'avantage de cette méthode est qu'elle permet de traiter le cas des mobiles d'agitation à géométries complexes. Le fichier contenant la liste des nœuds construits préalablement sur l'interface fluide-structure va être réutilisé au cours de l'exécution du code CFD afin de pouvoir spécifier les conditions aux limites au niveau de l'agitateur. Ce fichier apparaît ainsi d'une grande utilité puisqu'il propose une méthode universelle permettant d'étudier toutes les configurations à géométries complexes. Dans ces conditions, l'utilisateur n'aura plus besoin d'intervenir manuellement au niveau de notre programme pour pouvoir mettre à jour les équations et les adapter à la nouvelle géométrie, comme cela a été montré par Abid et al. [15], Baccar et al. [16-18] et Kchaou et al. [19]. Dans cette nouvelle démarche, les équations développées sont écrites dans une démarche standard, indépendante de la forme géométrique du mo- bile d'agitation choisi. Toutefois, on propose dans ces conditions d'utiliser des logiciels commercialisés de hautes performances pour une conception assistée par ordinateur. À côté du dessin de la géométrie, le logiciel CSD utilisé permet de créer un fichier de données contenant juste la liste des nœuds définissant le maillage du domaine solide. Ce fichier de données lorsque introduit dans notre interface graphique permet de générer l'ensemble des fichiers nécessaires pour l'exécution de notre code CFD. De ce fait, l'utilisation des logiciels CAO et CSD est d'une grande utilité. En effet, ces deux logiciels permettent de préparer tous les éléments nécessaires à l'utilisation de notre interface graphique et à l'exécution de notre code CFD. Toute cette démarche, après programmation, vient s'ajouter en pré-traitement à la résolution numérique des différentes équations, comme un nouveau module de maillage. Cette méthode permet d'apporter à notre code CFD plus de performance et d'efficacité afin de traiter de nouvelles géométries présentant des configurations complexes. Cette méthode a déjà été appliquée aux cas des turbines à pales droites [20], à pales incurvées [21] et à pales inclinées [12] en régime laminaire et turbulent. Les résultats trouvés se sont révélés satisfaisants. C'est pour cette raison que nous sommes amenés à appliquer cette même démarche numérique à des géométries beaucoup plus complexes tel que l'exemple traité dans cet article.

\section{Paramètres globaux}

L'opération d'agitation mécanique dépend de plusieurs paramètres globaux influant directement sur la qualité du produit obtenu. De ce fait, on est amené à présenter les différentes caractéristiques permettant de comparer les performances des agitateurs du point de vue consommation énergétique, qualité du mélange et capacité de pompage. La caractérisation d'un mobile d'agitation porte essentiellement sur la connaissance du nombre de puissance $N_{\mathrm{p}}$, du nombre de pompage $N Q_{\mathrm{p}}$, de l'efficacité de pompage $E_{\mathrm{p}}$ et de l'efficacité énergétique $E e$. Ces nombres caractéristiques varient en fonction du nombre de Reynolds $R e$ et des différents paramètres géométriques.

\subsection{Régime d'écoulement}

L'écoulement est défini par la vitesse du fluide et de sa direction dans la cuve. Cette vitesse exprime l'intensité de l'agitation dans la cuve et elle est déduite du débit créé par le mobile. L'intensité de l'agitation permet la détermination de l'aptitude du mobile à l'homogénéisation, à la dissolution, au transfert thermique et au macro mélange, c'est-à-dire aux applications nécessitant des courants de convection. Le régime d'écoulement peut être défini en spécifiant la valeur du nombre de Reynolds $R e$. Ce nombre adimensionnel est le rapport entre les forces d'inertie et les forces visqueuses mises en jeu lors de l'agitation. Selon la valeur du nombre de Reynolds $R e$, on peut distinguer le régime laminaire 
ou turbulent. En cuve agitée, le nombre de Reynolds Re est défini ainsi :

$$
R e=\frac{\rho N d^{2}}{\mu}
$$

\subsection{Nombre de puissance}

Dans une opération de mélange, lorsqu'un type de mobile d'agitation a été choisi, il reste à déterminer la puissance nécessaire à son entraînement. La puissance est un élément important puisqu'elle permet de choisir le type du moteur à installer. Elle est obtenue par intégration sur la surface de l'agitateur, de la puissance locale que le mobile transmet au fluide. Il est équivalent de dire que la puissance d'agitation est totalement transmise par le mobile au fluide au sein duquel elle est dissipée sous forme de chaleur [15]. En régime laminaire, la puissance $P$ s'écrit :

$$
P=\int_{v_{\mathrm{c}}} \mu \Phi_{\mathrm{v}} \mathrm{d} v
$$

$\mathrm{d} v$ représente un volume élémentaire de tout le volume de la cuve $v_{\mathrm{c}}$. Il s'exprime en repère cylindrique par la formule suivante :

$$
\mathrm{d} v=r \mathrm{~d} r \mathrm{~d} \theta \mathrm{d} z
$$

Le taux de dissipation visqueuse $\Phi_{\mathrm{v}}$ est défini par la formule suivante :

$$
\begin{aligned}
\Phi_{\mathrm{v}} & =\left[2\left[\left(\frac{\partial U}{\partial r}\right)^{2}+\left(\frac{\partial V}{r \partial \theta}+\frac{U}{r}\right)^{2}+\left(\frac{\partial W}{\partial z}\right)^{2}\right]\right. \\
& \left.+\left[\frac{\partial V}{\partial r}-\frac{V}{r}+\frac{\partial U}{r \partial \theta}\right]^{2}+\left[\frac{\partial W}{r \partial \theta}+\frac{\partial V}{\partial z}\right]^{2}+\left[\frac{\partial U}{\partial z}+\frac{\partial W}{\partial r}\right]^{2}\right]
\end{aligned}
$$

Une analyse dimensionnelle permet de faire apparaître un nombre adimensionnel appelé nombre de puissance $N_{\mathrm{p}}$. La connaissance de $N_{\mathrm{p}}$ permet ensuite d'effectuer les calculs d'extrapolation en puissance lorsque le diamètre $d$ et la vitesse de la turbine $N$ changent. En grandeur adimensionnelle, on peut définir le nombre de puissance par l'équation :

$$
N_{\mathrm{p}}=\frac{P}{\rho N^{3} d^{5}}
$$

\subsection{Nombre de pompage}

Le débit de pompage $Q_{\mathrm{p}}$ correspond au débit du fluide sortant du volume délimité par les dimensions du mobile d'agitation. Pour une turbine de diamètre $d$ et de hauteur $h$, l'expression générale du débit de pompage prend en compte le débit de pompage radial et le débit de pompage axial :

$$
Q_{\mathrm{p}}=\int_{z_{\mathrm{B}}}^{z_{\mathrm{B}}+h} \pi \mathrm{d}(U)_{r=d / 2} \mathrm{~d} z+\int_{0}^{d / 2} 2 \pi r(W)_{z=z_{\mathrm{B}}} \mathrm{d} r
$$

Dans cette équation, la première intégrale représente le débit de décharge dans le plan inférieur de l'agitateur et la seconde représente le débit radial positif sur le côté de l'agitateur. $z_{\mathrm{B}}$ est la position axiale situant le plan inférieur de l'agitateur et la coordonnée de la valeur maximale $z_{\mathrm{B}}+h$

Pour un agitateur radial, le débit de pompage ne prend en compte que le débit de fluide sortant radialement sur le côté :

$$
Q_{\mathrm{p}}=\int_{z_{\mathrm{B}}}^{z_{\mathrm{B}}+h} \pi \mathrm{d}(U)_{r=d / 2} \mathrm{~d} z
$$

Cependant, pour une turbine axiale il s'exprime par l'intégrale suivante :

$$
Q_{\mathrm{p}}=\int_{0}^{d / 2} 2 \pi r(W)_{z=z_{\mathrm{B}}} \mathrm{d} r
$$

En grandeur adimensionnelle, on peut définir le nombre de pompage par l'équation :

$$
N Q_{\mathrm{p}}=\frac{Q_{\mathrm{p}}}{N d^{3}}
$$

\subsection{Efficacité d'une turbine}

L'énergie dissipée par une turbine se transforme en cisaillement. De nombreux auteurs ont voulu quantifier la part de cisaillement dans l'énergie dissipée par un agitateur en définissant différentes efficacités à partir du nombre de pompage et du nombre de puissance.

\subsubsection{Efficacité de pompage}

Weetman et Oldshue [22] ont comparé les rapports $Q_{\mathrm{p}} / P$ pour diverses turbines et ont constaté que les turbines axiales sont plus efficaces que les turbines radiales en terme de débit de fluide par unité de puissance. L'efficacité de pompage $E_{\mathrm{p}}$ est définie par l'expression suivante :

$$
E_{\mathrm{p}}=\frac{N Q_{\mathrm{p}}}{N_{\mathrm{p}}}
$$

Une turbine à forte valeur de $E_{\mathrm{p}}$ génère plus de mouvements de convection et sera alors plus adaptée à des applications contrôlées par la convection, comme par exemple le mélange de fluides miscibles.

\subsubsection{Efficacité énergétique}

Medek et Fort [23] ont défini l'efficacité énergétique Ee de l'agitateur par la relation suivante :

$$
E e=\frac{N Q p^{3}}{N_{\mathrm{p}}}
$$




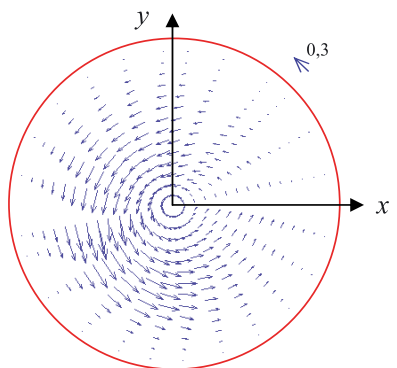

(a) Mono vis

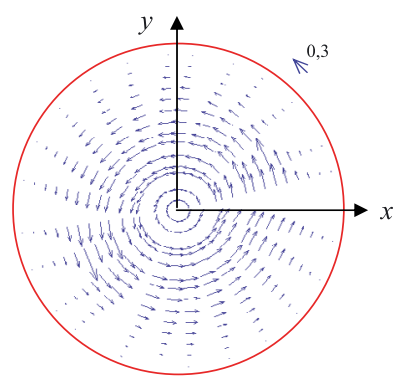

(c) Double vis

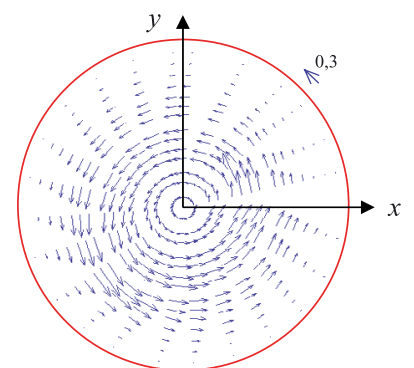

(b) Mono vis modifiée

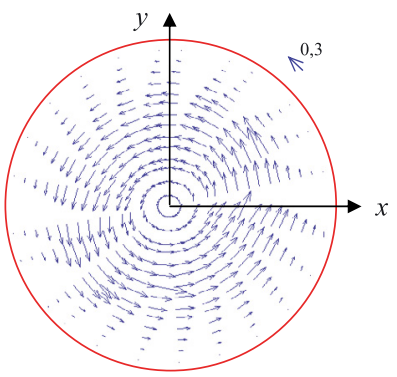

(d) Double vis modifiée
Fig. 4. Champs des vitesses dans le plan horizontal défini par $z=1$.

L'efficacité énergétique exprime le besoin en puissance d'un agitateur pour fournir un débit de pompage donné. Pour deux agitateurs différents, le rapport des efficacités énergétiques est inversement proportionnel au rapport des puissances consommées par chaque agitateur à un débit de pompage constant. Cette expression de l'efficacité est la plus adaptée pour comparer les performances relatives de différents types de turbines d'agitation.

\section{Résultats numériques}

Les résultats numériques locaux présentés dans ce paragraphe correspondent à un régime d'écoulement laminaire défini par un nombre de Reynolds $R e=4$ et un nombre de Froude $F_{r}=0,087$.

\subsection{Champ des vitesses}

\subsubsection{Plan horizontal $r-\theta$}

Sur la figure 4 est présentée le champ des vitesses de la résultante des composantes radiale et tangentielle de la vitesse dans le plan horizontal $r-\theta$ situé à une position axiale $z=1$. Ces résultats numériques correspondent aux quatre configurations déjà définies précédemment à savoir les mono et les doubles vis à profil simple et modifié. Ces résultats montrent que l'amplitude des vitesses est plus importante au niveau de la vis avec dominance d'un écoulement tangentiel dû à la mise en rotation du volume balayé par les turbines. Loin de ce domaine, on note un ralentissement progressif de l'écoulement. Par ailleurs, on constate que le nombre des vis ainsi que la forme du profil

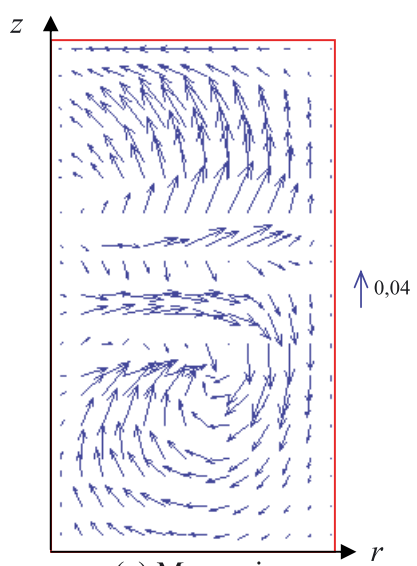

(a) Mono vis

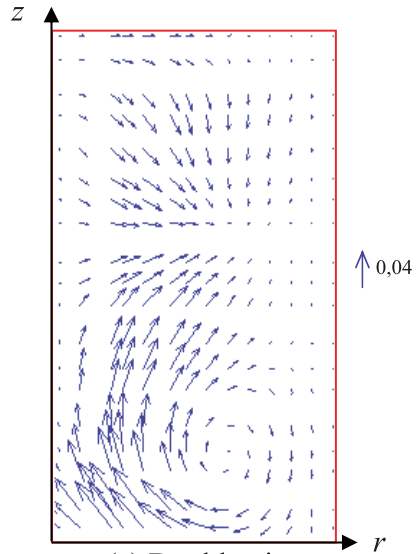

(c) Double vis

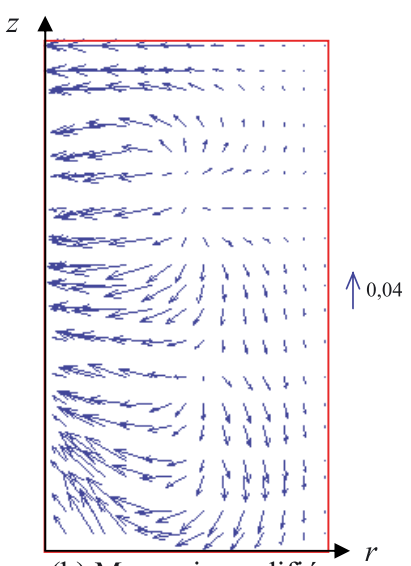

(b) Mono vis modifiée

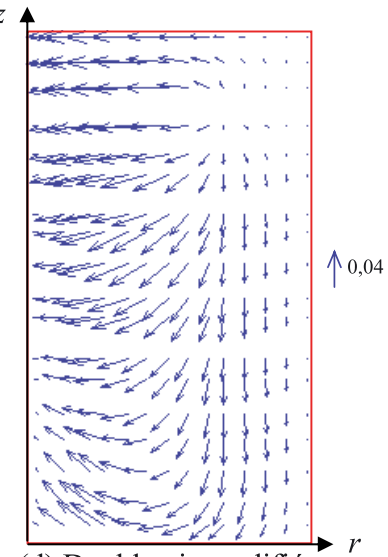

(d) Double vis modifiée
Fig. 5. Champs des vitesses dans le plan vertical défini par $\theta=116^{\circ}$.

jouent un rôle déterminant dans la présentation du champ des vitesses. En effet, avec une double vis on constate que le champ des vitesses est très actif des deux côtés opposés qui correspondent à la zone de contact du fluide avec la surface d'attaque du mobile d'agitation. Également, on note que le champ des vitesses pour le cas du mono et du double vis à profil modifié est plus actif que les configurations à profil simple.

\subsubsection{Plans verticaux $r-z$}

Sur les figures 5 et 6 est présentée la distribution du champ des vitesses de la résultante des composantes radiales et axiales dans les deux plans verticaux $r-z$ correspondant respectivement à des positions angulaires de la cuve égales à $\theta=116^{\circ}$ et $\theta=331^{\circ}$. Ces positions ont été choisies afin de montrer les variations que présente le champ des vitesses dans le plan de présentation $r-z$. Avec une mono vis à profil simple, on constate que l'écoulement apparaît à dominance axiale. Cet écoulement est ascendant en haut de la cuve et descendant en bas de la cuve. Également, on rencontre un écoulement radial juste dans les zones restreintes situées au fond, à mi-hauteur et juste en haut de la cuve (Fig. 5a). Ces résultats sont dictés par la forme géométrique de la vis. Avec une double vis, cette 


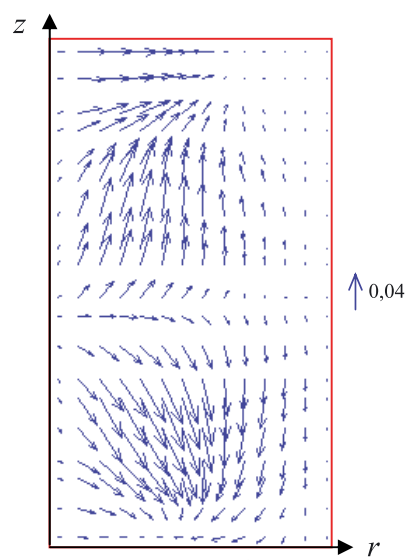

(a) Mono vis

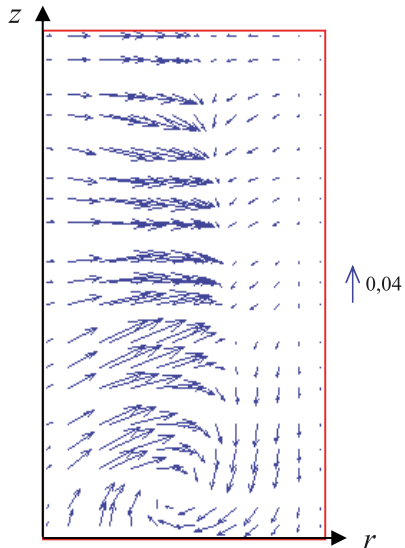

(c) Double vis

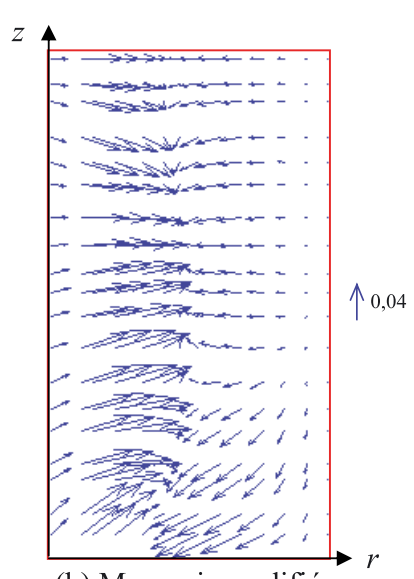

(b) Mono vis modifiée

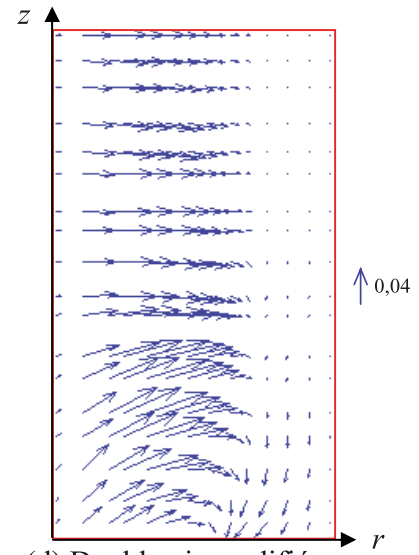

(d) Double vis modifiée

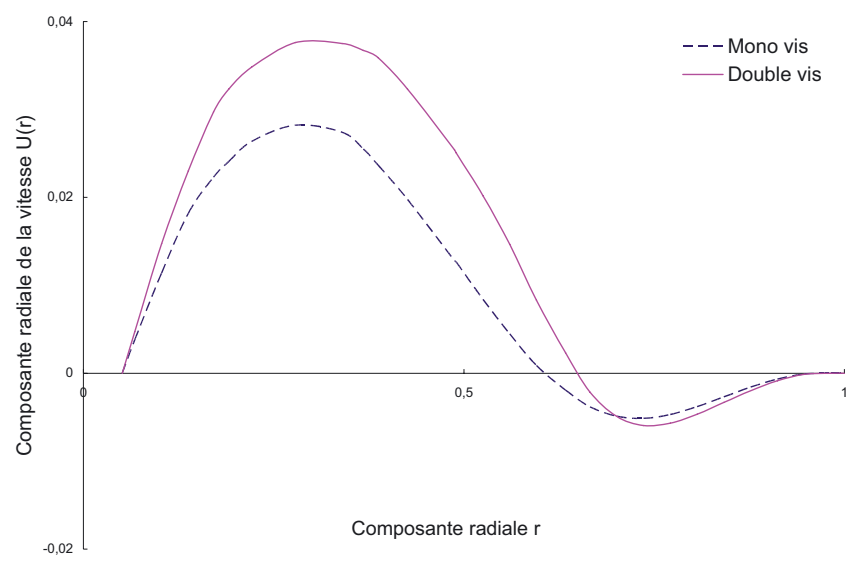

(a) Plan horizontal défini par $z=0,45$

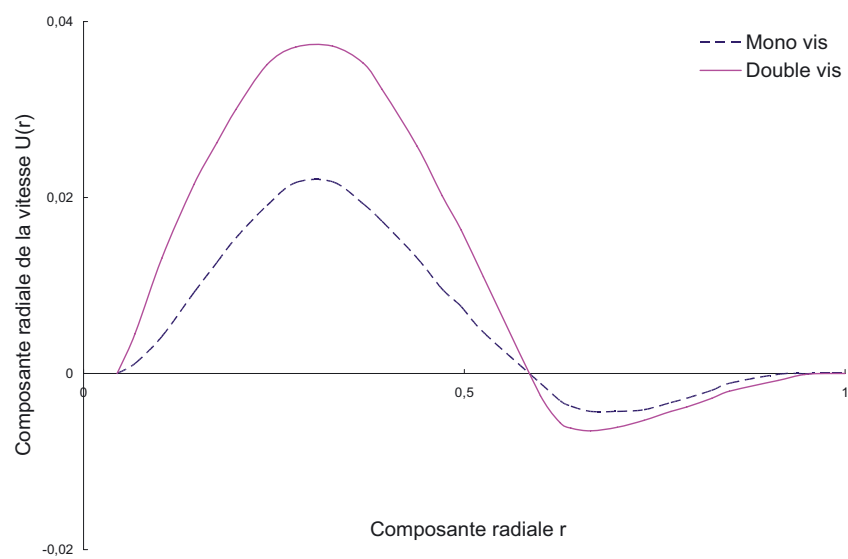

(b) Plan horizontal défini par $z=0,8$

Fig. 7. Variation radiale de la vitesse radiale $U(r)$.

on constate qu'à côté de l'axe l'écoulement s'inverse et devient de type centrifuge (Fig. 6d). À côté des parois, l'écoulement est plutôt radial en haut de la cuve et oblique juste en bas. En analysant ces résultats trouvés dans le cas d'une vis à profil modifié, on constate la disparition des boucles de recirculation déjà trouvées dans le cas d'une vis à profil simple.

\subsection{Profils radiaux de la vitesse}

Sur les figures 7-9 sont comparés les résultats issus de l'application de notre code de calcul pour le cas d'une mono vis et d'une double vis. Plus particulièrement, on s'est intéressé à la comparaison des courbes présentant l'évolution radiale des composantes radiale $U(r)$, tangentielle $V(r)$ et axiale $W(r)$ de la vitesse. Ces profils radiaux présentés appartiennent à deux plans horizontaux différents définis par les positions axiales égales à $z=0,45$ et $z=0,8$ de la cuve agitée. D'après la figure 7 , on constate que la composante radiale $U(r)$ de la vitesse atteint sa valeur maximale égale à $U=0,039$. La position radiale correspondante à cette valeur maximale est définie par $r=0,28$. Cette même allure présente une vitesse minimale $U=-0,005$ à une position radiale $r=0,7$. l'adjonction d'une deuxième vis améliore le mouvement du fluide (Fig. 5d). Pour la deuxième position angulaire,
A côté des parois, l'écoulement est plutôt axial pompant vers le bas de la cuve (Fig. 5b). Avec une double vis modifiée, le même phénomène est également observé pour ces différents plans. Toutefois, l'intensité de la vitesse est plus importante dans ce cas. Ce résultat est déjà attendu, car 


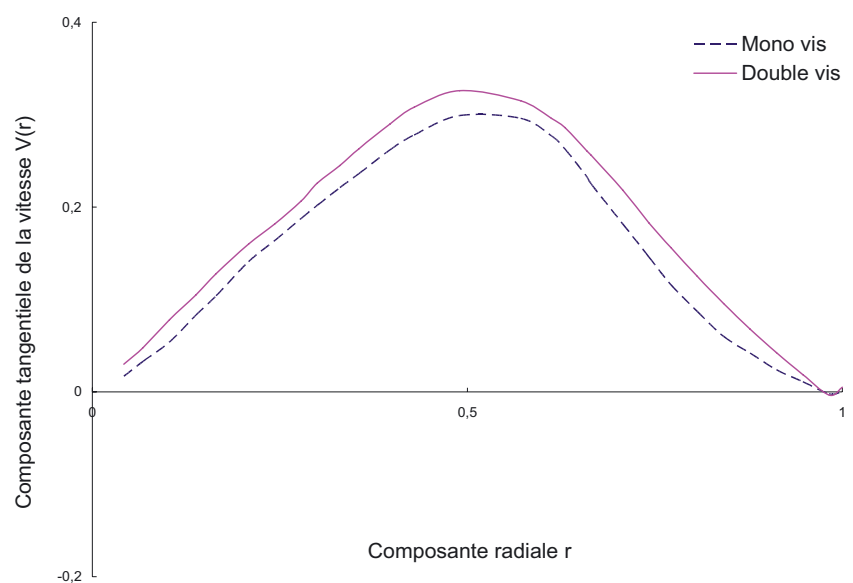

(a) Plan horizontal défini par $z=0,45$

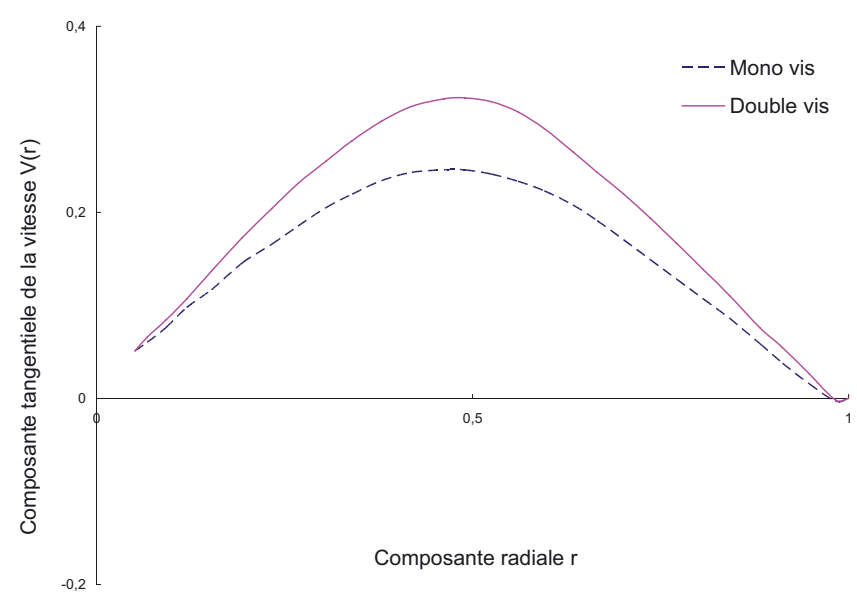

(b) Plan horizontal défini par $z=0,8$

Fig. 8. Variation radiale de la vitesse tangentielle $V(r)$.

De ce fait, en s'approchant de l'axe de rotation du mobile d'agitation, l'écoulement est de type centrifuge. Il devient centripète en s'approchant des parois de la cuve. La composante tangentielle $V(r)$ de la vitesse présente une allure parabolique (Fig. 8). Son extremum est défini par une position radiale $r=0,5$. Dans cette position, la composante tangentielle de la vitesse atteint une valeur égale à $V=0,34$. Au voisinage de l'axe de rotation du mobile d'agitation et des parois de la cuve, cette composante atteint des valeurs très faibles. D'après la figure 9 , on constate que l'allure de la composante axiale de la vitesse $W(r)$ présente deux extrema. La position radiale de cet extremum est largement affectée par la position axiale du plan de présentation de la cuve. En effet, dans le plan horizontal situé à une position axiale $z=0,45$, la valeur maximale de la composante axiale $W(r)$ atteint une valeur égale à $W=0,035$ pour une position radiale $r=0,4$. Toutefois, dans le plan horizontal défini par $z=0,8$, cette position radiale devient égale à $r=0,65$. La valeur positive de la composante axiale $W(r)$ de la vitesse correspond à un mouvement ascendant de l'écoulement vers la surface libre de la cuve. Également, on note que dans le plan horizontal situé à une position axiale $z=0,45$, la valeur

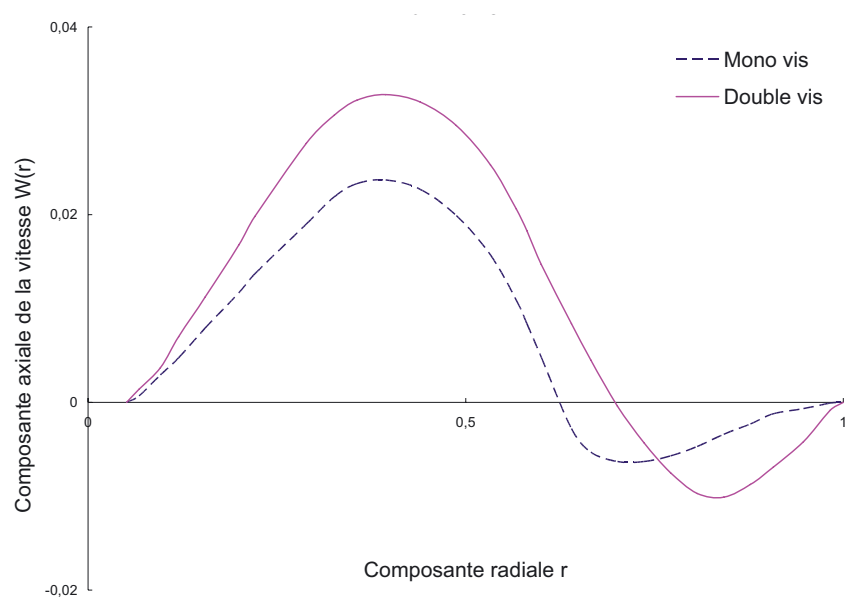

(a) Plan horizontal défini par $z=0,45$

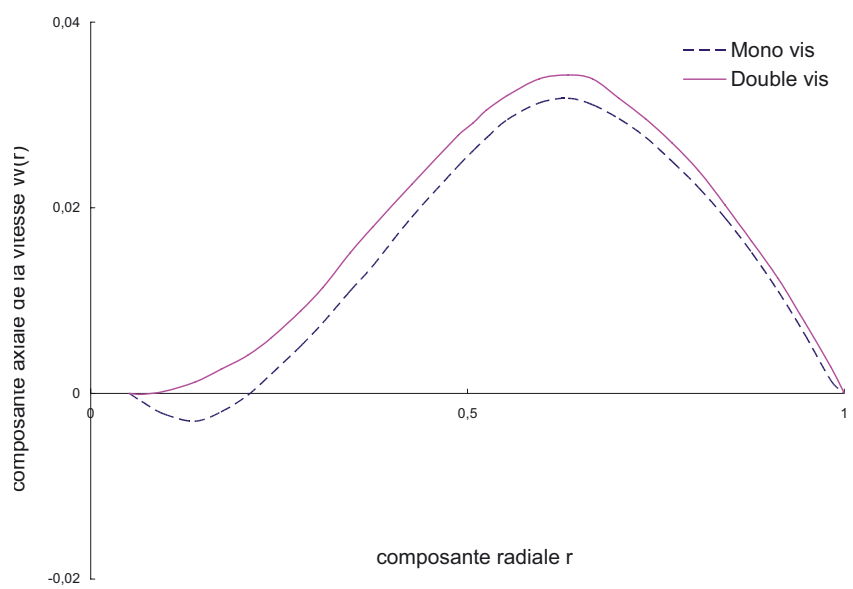

(b) Plan horizontal défini par $z=0,8$

Fig. 9. Variation radiale de la vitesse axiale $W(r)$.

minimale de la composante axiale $W(r)$ atteint une valeur égale à $W=-0,01$ pour une position radiale $r=0,85$ (Fig. 9a). Cette valeur devient égale à $W=-0,004$ pour une position radiale $r=0,12$ (Fig. 9b). La valeur négative de la composante axiale $W(r)$ correspond à un mouvement descendant vers le fond de la cuve. Globalement, on constate que la double vis possède un champ de vitesse plus actif que celle de la mono vis. Par ailleurs, ces résultats confirment les constatations déjà signalées auparavant.

\subsection{Taux de dissipation visqueuse}

Sur les figures 10 et 11 est présentée la répartition du taux de dissipation visqueuse dans différents plans horizontaux $r-\theta$. Ces plans de présentation sont situés respectivement à des positions axiales définies par $z=0,95$ et $z=1,15$. D'autres plans de présentation sont également considérés; ils correspondent à différents plans verticaux $r-z$ définis par les positions angulaires $\theta=108^{\circ}$ et $\theta=259^{\circ}$ (Figs. 12 et 13 ). Globalement, on constate que la région siège des valeurs maximales se localise dans le 


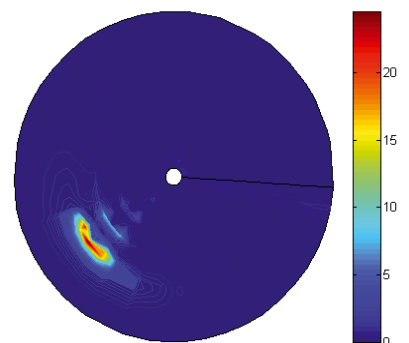

(a) Mono vis

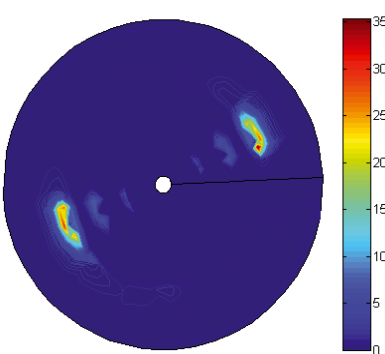

(c) Double vis

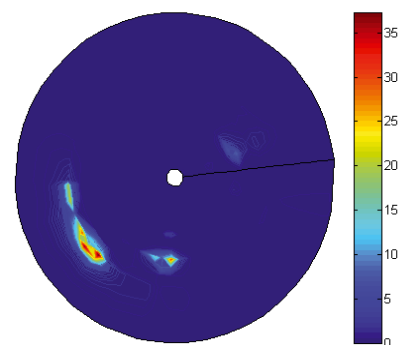

(b) Mono vis modifiée

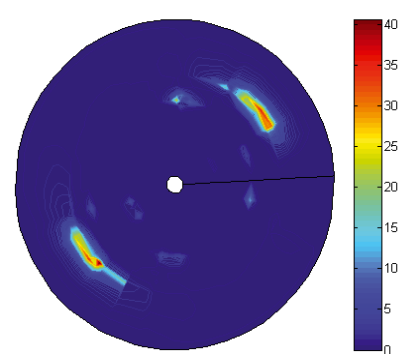

(d) Double vis modifiée
Fig. 10. Répartition du taux de dissipation dans le plan horizontal défini par $z=0,95$.

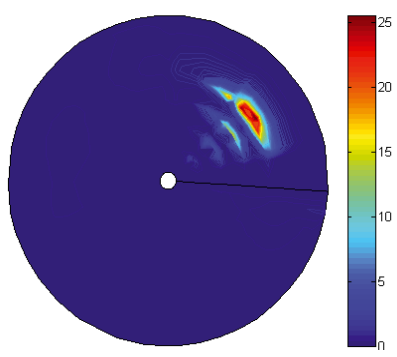

(a) Mono vis

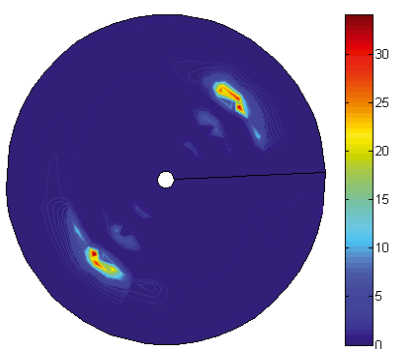

(c) Double vis

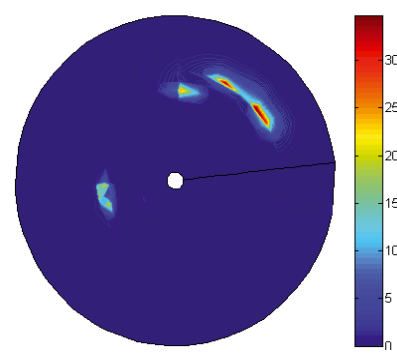

(b) Mono vis modifiée

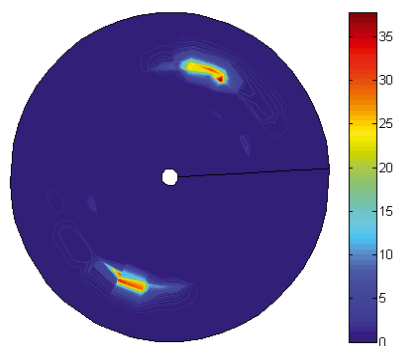

(d) Double vis modifiée
Fig. 11. Répartition du taux de dissipation dans le plan horizontal défini par $z=1,15$.

sillage qui se développe au niveau du bout de la vis. Hors de ce domaine, le taux de dissipation devient rapidement très faible. D'après ces résultats, on constate que la forme du profil de la vis a une influence sur le taux de dissipation visqueuse. Les valeurs les plus grandes du taux de dissipation sont atteintes dans le cas d'une vis à profil modifié (Figs. 12b, 13b, 12d et 13d). De même, on vérifie

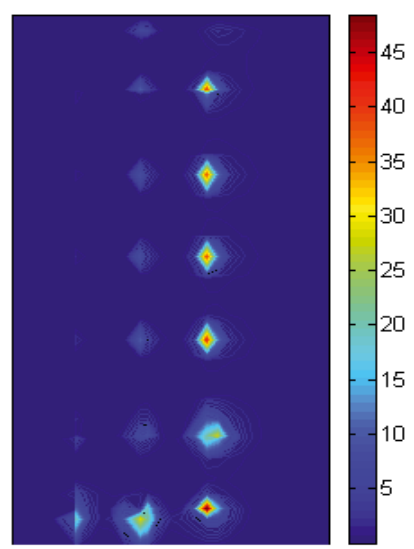

(a) Mono vis

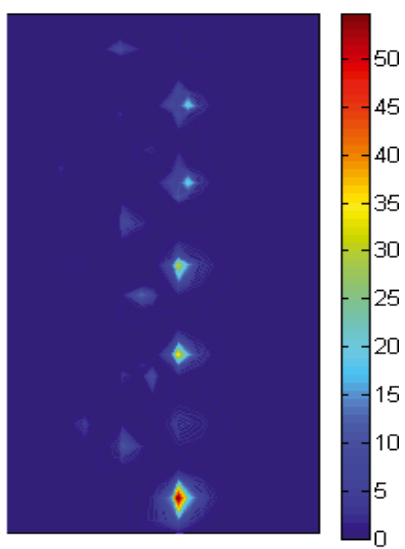

(b) Mono vis modifiée (c) Double vis

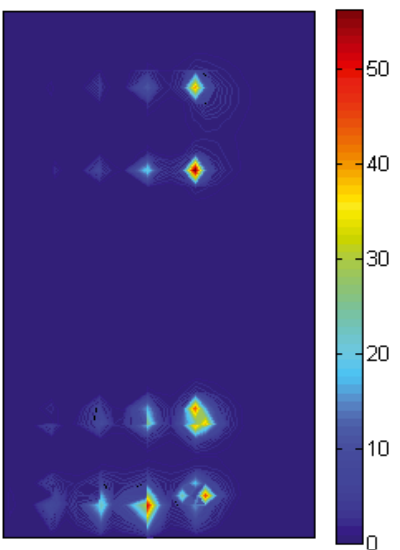

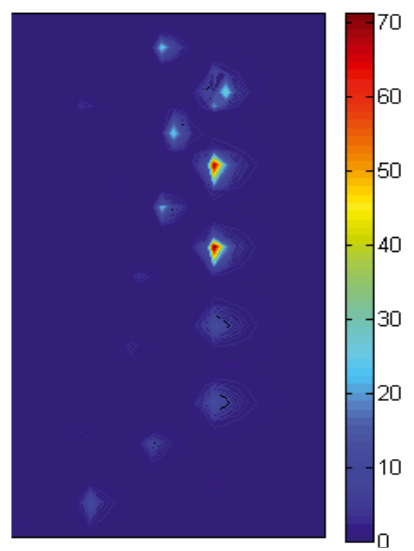

(d) Double vis modifiée
Fig. 12. Répartition du taux de dissipation dans le plan défini par $\theta=108^{\circ}$.

qu'une double vis présente une dissipation visqueuse plus importante que celle d'une mono vis. Dans ces conditions, on assiste à l'apparition d'une deuxième zone siège des valeurs maximales du taux de dissipation visqueuse située à $180^{\circ}$ par rapport à la première zone. Ce résultat est déjà attendu vue la symétrie que présente la géométrie d'une double vis.

\subsection{Caractéristiques globales}

Sur les figures 14-16 est présentée la variation du nombre de pompage $N Q_{\mathrm{p}}$, de l'efficacité de pompage $E_{\mathrm{p}}$ et de l'efficacité énergétique $E e$ en fonction du nombre de Reynolds $R e$ dans le cas d'une mono et d'un double vis. D'après ces résultats, on constate que ces trois paramètres présentent des fonctions croissantes avec l'augmentation du nombre de Reynolds. En échelle logarithmique, ils suivent une allure presque linéaire avec des pentes positives. Globalement, on constate que pour une double vis l'effet de pompage est plus important que dans le cas d'une mono vis. L'efficacité de pompage et 


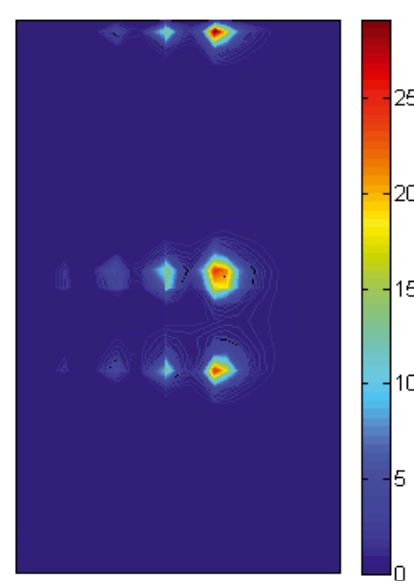

(a) Mono vis

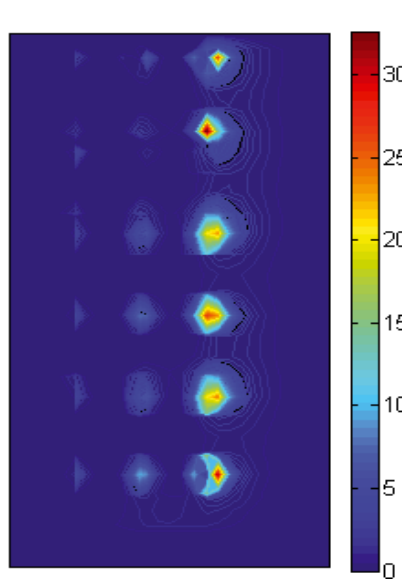

(c) Double vis

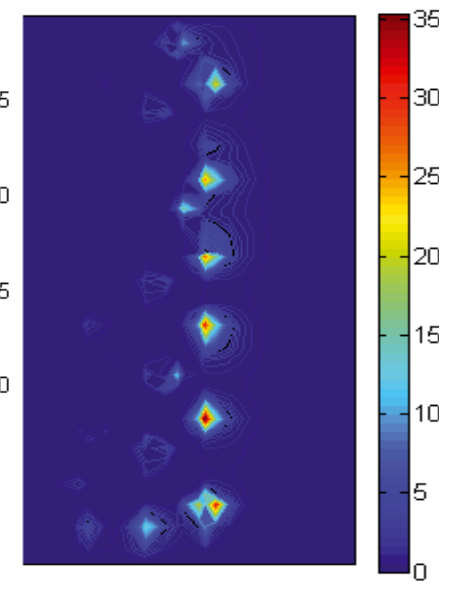

(b) Mono vis modifiée

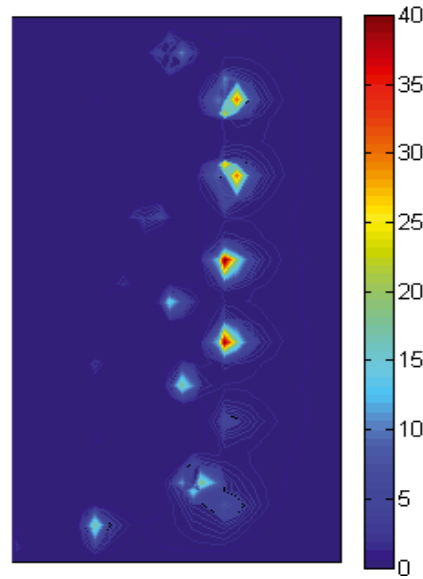

(d) Double vis modifiée
Fig. 13. Répartition du taux de dissipation dans le plan défini par $\theta=259^{\circ}$.

l'efficacité énergétique atteignent également les valeurs les plus grandes dans le cas d'une double vis.

\section{Comparaison avec des résultats antérieurs}

Sur la figure 17 est présentée la variation du nombre de puissance $N_{\mathrm{p}}$ en fonction du nombre de Reynolds $R e$ pour le cas d'une mono et d'une double vis. Plus particulièrement, on a superposé les résultas numériques issus de l'application de notre code de calcul CFD et les résultats expérimentaux obtenus par Nagata [1]. D'après ces résultats, on remarque que ces courbes présentent une même allure. En échelle logarithmique, cette allure présente une fonction linéaire décroissante. Les valeurs de la constante de la puissance $K_{\mathrm{p}}=N_{\mathrm{p}} R e$ relatives au cas d'une mono vis et d'une double vis sont égales respectivement à 438 et à 600 . Toutefois, on constate une légère différence entre les deux résultats. En s'intéressant aux résultats relatifs au cas d'une mono vis, on constate que le nombre de puissance calculé à l'aide de notre code de

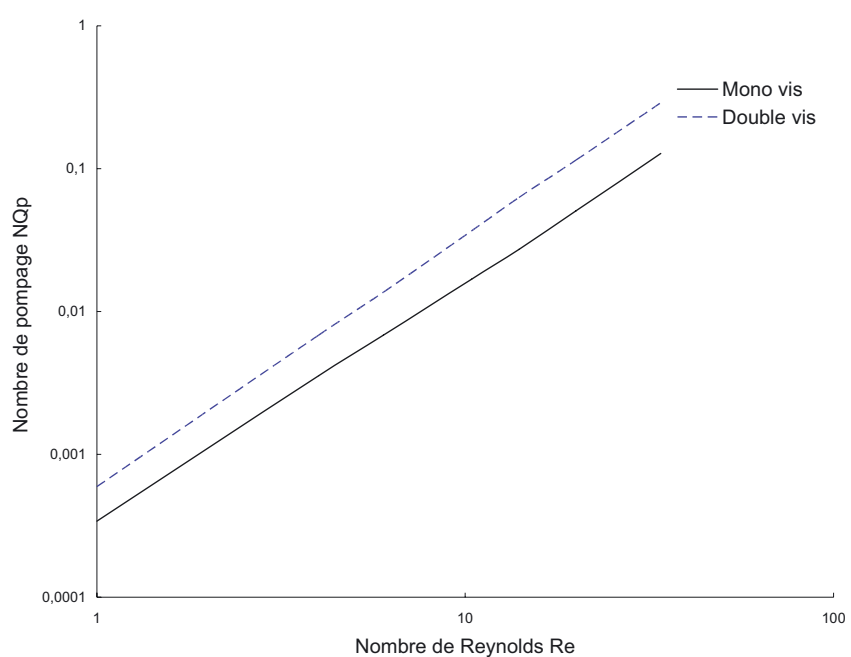

Fig. 14. Variation de $N Q_{\mathrm{p}}=f(R e)$.

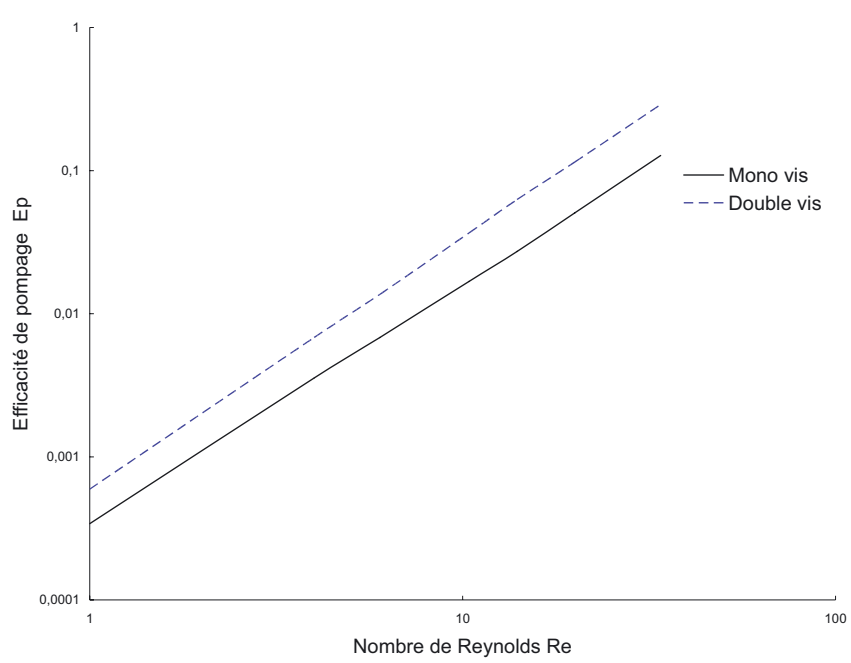

Fig. 15. Variation de $E_{\mathrm{p}}=f(R e)$.

calcul est légèrement supérieur aux résultats expérimentaux tirés de la littérature [1]. En calculant l'écart relatif entre les deux résultats numérique et expérimental, on trouve une variation de l'ordre de $10 \%$. Cet écart est raisonnable et permet de justifier la méthode d'analyse adoptée.

\section{Conclusion}

En vue de mettre en exergue les performances des systèmes d'agitation mécanique, un code de simulation tridimensionnel a été développé. La procédure de résolution numérique et les différentes techniques utilisées pour la mise au point de ce code sont également présentées. Ces développements apportés à notre code sont faits afin de pouvoir développer une démarche standard permettant l'étude de la structure hydrodynamique générée par différents types de mobiles d'agitation. Cette démarche de résolution numérique présente une grande maniabilité dans le traitement des conditions aux limites 


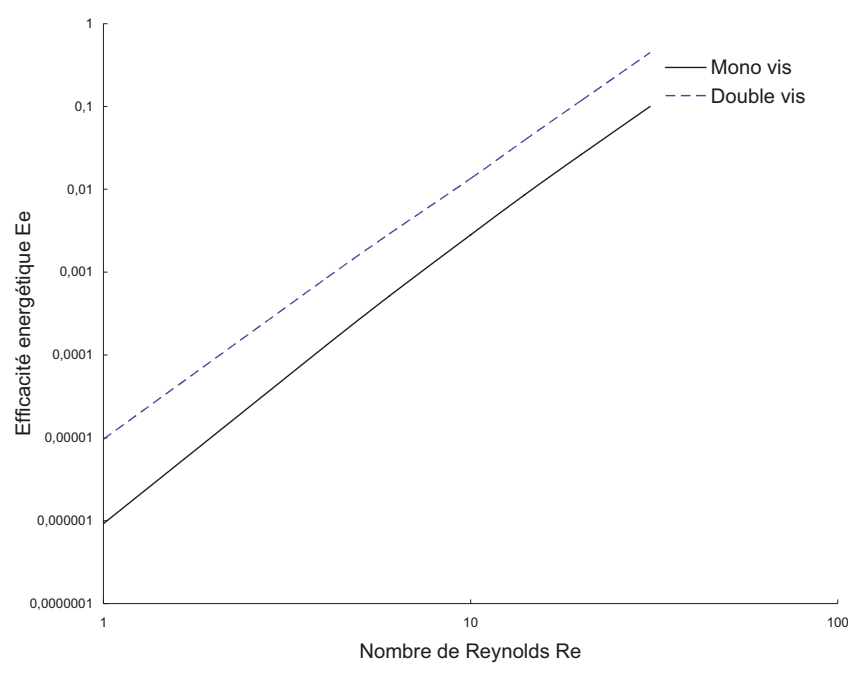

Fig. 16. Variation de $E_{\mathrm{e}}=f(R e)$.

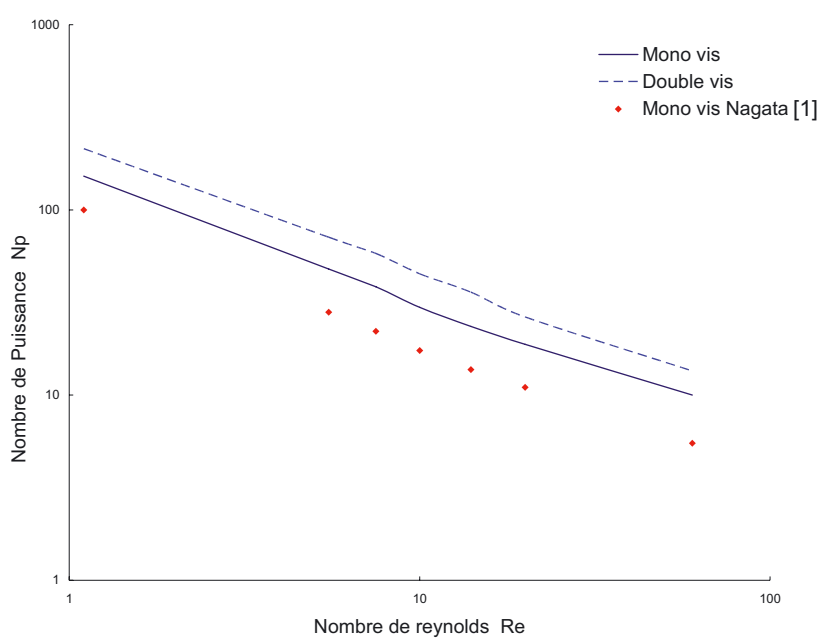

Fig. 17. Variation de $N_{\mathrm{p}}=f(R e)$.

surtout dans le cas des géométries complexes. Dans cet article, nous nous sommes intéressés à l'analyse de la structure hydrodynamique générée par les mobiles de proximité type mono et double vis avec profil simple et modifié. Pour ces mobiles d'agitation, on a présenté la distribution du champ des vitesses et du taux de dissipation dans différents plans de la cuve agitée ainsi que ses différentes caractéristiques globales. Le but a été d'étudier l'influence du nombre de vis ainsi que la forme du profil sur la structure hydrodynamique de la cuve. Les résultats numériques nous ont permis d'apporter des informations précises sur les conditions géométriques optimales de l'écoulement généré par ces différentes configurations. En effet, nous avons montré dans ce travail que le nombre de vis ainsi que la forme du profil jouent un rôle déterminant dans la présentation du champ des vitesses. Plus particulièrement, on a constaté que le champ des vitesses dans le cas d'une vis à profil modifié est plus actif que les configurations à profil simple. Globalement, on a constaté que pour une double vis l'effet de la dissipation visqueuse et du pompage est plus important que dans le cas d'une mono vis. Également, on a montré dans ce cas que l'efficacité de pompage et l'efficacité énergétique atteignent les valeurs les plus grandes.

Les résultats numériques trouvés peuvent fournir un appui indispensable à des nouvelles avancées technologiques. Cela permet éventuellement d'aider à la décision pour un choix adéquat des équipements industriels.

\section{Références}

[1] S. Nagata, Mixing: principles and applications, Halstead press, Japan, 1975

[2] J. Aubin, D.F. Fletcher, C. Xuereb, Modeling turbulent flow in stirred tanks with CFD: the influence of the modeling approach, turbulence model and numerical scheme, Exp. Th. Fluid Sci. 28 (2004) 431-445

[3] M. Mseddi, Z. Driss, M. Baccar, M.S. Abid, Étude d'une soupape de décharge de turbocompresseur de suralimentation, Mécanique \& Industries 6 (2005) 539-551

[4] Abdelkader Noureddine, Écoulement turbulent entre un disque tournant et un disque fixe, influence des phénomènes d'entrée liés à la valeur finie des rayons, Mécanique \& Industries 10 (2009) 421-428

[5] R. Valette, J. Bruchon, H. Digonnet, P. Laure, M. Leboeuf, L. Silva, B. Vergnes, T. Coupez, Méthodes d'interaction fluide-structure pour la simulation multiéchelles des procédés de mélange, Mécanique \& Industries 8 (2007) 251-258

[6] G. Delaplacea, R. Guerina, J.C. Leulieta, R.P. Chhabrab, An analytical model for the prediction of power consumption for shear-thinning fluids with helical ribbon and helical screw ribbon impellers, Chem. Eng. Sci. 61 (2006) 3250-3259

[7] F. Bertrand, F. Thibault, L. Delamare, P.A. Tanguy, Adaptive finite element simulations of fluid flow in twinscrew extruders, Comput. Chem. Eng. 27 (2003) 491-500

[8] B.J. Bellhouse, G. Costigan, K. Abhinava, A. Merry, The performance of helical screw-thread inserts in tubular membranes, Separation and Purification Technology 2223 (2001) 89-113

[9] F. Rieger, Pumping efficiency of screw agitators 3 in a tube, Chem. Eng. J. 89 (2002) 47-52

[10] C. Rorres, The turn of the screw: Optimal design of an Archimedes screw, J. Hydraul. Eng. 126 (2000) 72-80

[11] Z. Driss, Contribution à l'étude des turbines en cuves agitées, Thèse, Université de Sfax, École Nationale d'Ingénieurs de Sfax, 2008

[12] Z. Driss, G. Bouzgarrou, W. Chtourou, H. Kchaou, M. S. Abid, Computational studies of the pitched blade turbines design effect on the stirred tank flow characteristics, Eur. J. F. Mech B, Fluids 29 (2010) 236-245

[13] S.V. Patankar, Numerical heat transfer and fluid flow, Series in Computational Methods in Mechanics and Thermal Sciences, Mc Graw Hill, New York, 1980 
[14] J. Douglas, J.E. Gunn, A general formulation of alternating direction implicit methods, Num. Math. 6 (1964) $428-453$

[15] M.S. Abid, C. Xuereb, J. Bertrand, Modeling of the 3D hydrodynamics of 2-blade impellers in stirred tanks filled with a highly viscous fluid, Canadian J. Chem. Eng. 72 (1994) 184-193

[16] M. Baccar, H. Kchaou, M. Mseddi, M.S. Abid, Modélisation de l'écoulement turbulent induit par une turbine de Rushton en standard : approche pseudostationnaire, Mécanique \& Industries 4 (2003) 301-318

[17] M. Baccar, M.S. Abid, Simulation numérique des comportements hydrodynamiques et thermiques des échangeurs racleurs opérant en régime turbulent, Int. J. Therm. Sci. 38 (1999) 634-644

[18] M. Baccar, M.S. Abid, Simulation numérique des comportements hydrodynamique et thermique des produits newtonien et rhéofluidifiant en cuve agitée par des mobiles à ancres et barrières, Entropie 36 (2000) 22-29
[19] H. Kchaou, M. Baccar, M. Mseddi, M.S. Abid, Modélisation numérique de l'écoulement interne turbulent généré par une turbine radiale en cuve agitée, Revue Française de Mécanique 1 (2000) 73-79

[20] Z. Driss, H. Kchaou, M. Baccar, M.S. Abid, Numerical investigation of internal laminar flow generated by a retreated-blade paddle and a flat-blade paddle in a vessel tank, Int. J. Eng. Sim. 6 (2005) 10-16

[21] Z. Driss, S. Karray, H. Kchaou, M.S. Abid, Computer simulations of fluid-structure interaction generated by a flat-blade paddle in a vessel tank, Int. Rev. Mech. Eng. 6 (2007) 608-617

[22] R.J. Weetman, J.Y. Oldshue, Power flow and shear characteristics of mixing impellers, in BHRA Proc. 6th. Europ. Mixing Conf., Pavia, Italy, 1988, pp. 43-50

[23] J. Medek, I. Fort, Mixing in vessel with eccentrical mixer, in BHRA Proc. 5th. Europ. Mixing Conf., Wurzburg, Germany, 1985, pp. 263-271 